\title{
Osteossíntese de fraturas do colo do metacarpo com parafuso autocompressivo - Análise preliminar de 21 casos $^{*}$
}

\section{Osteosynthesis of Fractures of the Metacarpal Neck with Self-Compressing Screw - Preliminary Analysis of 21 Cases}

\author{
Celso Ricardo Folberg ${ }^{10}$ Jairo André de Oliveira Alves ${ }^{10}$ Otávio Pereira Cadore ${ }^{2(1)}$ \\ Fernando Maurente Sirena10
}

\footnotetext{
${ }^{1}$ Grupo de Cirurgia da Mão, Serviço de Ortopedia e Traumatologia, Hospital de Clínicas de Porto Alegre, Porto Alegre, RS, Brasil

2 Serviço de Ortopedia e Traumatologia, Hospital de Clínicas de Porto Alegre, Porto Alegre, RS, Brasil
}

\author{
Endereço para correspondência Celso Ricardo Folberg, MSc, Rua \\ Sinke, 236, casa 45, Porto Alegre, RS, 90840-150, Brasil \\ (e-mail: cfolberg@hcpa.edu.br).
}

Rev Bras Ortop 2021;56(2):198-204.

\begin{abstract}
Resumo
Objetivo O presente estudo visa analisar os resultados clínicos do tratamento cirúrgico das fraturas de colo do metacarpo com fixação intramedular retrógrada utilizando parafusos canulados sem cabeça (tipo Herbert).

Métodos Estudo retrospectivo de 21 fraturas fechadas desviadas do colo do metacarpo em 21 pacientes operados entre abril de 2015 e novembro de 2018.

Resultados A casuística incluiu 19 homens e 2 mulheres. Os mecanismos causadores do trauma foram soco, queda ao solo e acidente com veículo motorizado ( $n=14,5$ e 2). Os metacarpos acometidos foram o V, III e II ( $n=19,1$ e 1$)$. As indicações cirúrgicas foram angulação colo-diáfise do metacarpo $>30^{\circ}$ para os II e III metacarpos e $>40^{\circ}$ para o $\vee$ metacarpo, encurtamento $\geq 5 \mathrm{~mm}$, desvio rotacional e o desejo do paciente de

\section{Palavras-chave}

- fraturas ósseas

- mãos

- metacarpo não utilizar imobilização gessada. No pós-operatório imediato, os pacientes permaneceram sem imobilização e orientados a mobilizar os dedos conforme tolerância. Todos os pacientes ficaram com mobilidade ativa total $>240^{\circ}$ e retornaram às suas antigas ocupações. Todas fraturas consolidaram e não houve reintervenções.
\end{abstract}

\footnotetext{
Trabalho desenvolvido no Grupo de Cirurgia da Mão, Serviço de Ortopedia e Traumatologia, Hospital de Clínicas de Porto Alegre (HCPA), Porto Alegre, RS, Brasil.
}

recebido

17 de Janeiro de 2020

aceito

05 de Maio de 2020
DOI https://doi.org/ 10.1055/s-0040-1714229. ISSN 0102-3616.

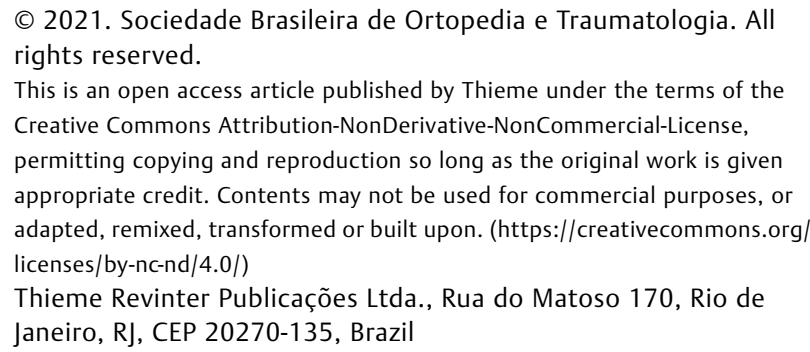

(c) 2021. Sociedade Brasileira de Ortopedia e Traumatologia. All rights reserved.

This is an open access article published by Thieme under the terms of the Creative Commons Attribution-NonDerivative-NonCommercial-License, permitting copying and reproduction so long as the original work is given appropriate credit. Contents may not be used for commercial purposes, or adapted, remixed, transformed or built upon. (https://creativecommons.org/ licenses/by-nc-nd/4.0/) Thieme Revinter Publicações Ltda., Rua do Matoso 170, Rio de Janeiro, RJ, CEP 20270-135, Brazil 


\section{Abstract \\ Keywords \\ - fractures, bone \\ - hand \\ - metacarpus}

Discussão As grandes vantagens da técnica com parafuso sem cabeça são sua baixa morbidade, estabilidade suficiente para não precisar de imobilização externa e reprodutibilidade com baixo custo.

Conclusão Esta é uma técnica fácil, rápida, e que apresenta ótimos resultados para o tratamento cirúrgico das fraturas deslocadas do colo dos metacarpos.

Objective The present study aims to analyze the clinical results of the surgical treatment of metacarpal neck fractures with retrograde intramedullary fixation using cannulated headless screws (Herbert type).

Methods Retrospective study of 21 closed fractures deviated from the metacarpal neck in 21 patients operated between April 2015 and November 2018.

Results The sample included 19 men and 2 women. The mechanisms that caused the trauma were punching, falling to the ground and motor vehicle accident $(n=14,5$ and $2)$. The affected metacarpals were the $5^{\text {th }}, 3^{\text {rd }}$, and $2^{\text {nd }}(n=19,1$ and 1$)$. Surgical indications were neck-shaft diaphysis of the metacarpal $>30^{\circ}$ for the $2^{\text {nd }}$ and $3^{\text {rd }}$ metacarpals and $>40^{\circ}$ for the $5^{\text {th }}$ metacarpal, shortening $\geq 5 \mathrm{~mm}$, rotational deviation, and the desire of the patient not to use plaster cast. In the immediate postoperative period, patients remained without immobilization and were instructed to mobilize their fingers according to tolerance. All patients had total active mobility $>240^{\circ}$ and returned to their former occupations. All fractures consolidated and there were no reinterventions.

Discussion The great advantages of the headless screw technique are its low morbidity, sufficient stability to avoid external immobilization, and reproducibility at low cost.

Conclusion This is an easy, fast technique that has excellent results for the surgical treatment of displaced fractures of the neck of the metacarpals.

\section{Introdução}

Fraturas de metacarpos e falanges correspondem a $18 \%$ de todas as fraturas abaixo do cotovelo, ${ }^{1}$ e podem ser tratadas de diversas maneiras. Fraturas do colo dos metacarpos podem ter indicação cirúrgica ao apresentar desvio rotacional, angulação ou encurtamento, sendo que os parâmetros exatos do alinhamento aceitável ainda são controversos. ${ }^{2}$

Dentre os métodos de fixação interna, podemos destacar fios de Kirschner, placas e parafusos. Os primeiros geralmente exigem imobilização pós-operatória, promovendo rigidez articular por não permitir mobilidade precoce. $^{3}$ Placas e parafusos geralmente requerem dissecção extensa de partes moles, podendo causar rigidez e aderências tendíneas. Embora não haja consenso ainda de qual é o melhor método, o tratamento cirúrgico abrange uma taxa geral de complicações de até $36 \%$. Destas, as mais comuns são: lesão tendínea, pseudoartrose, consolidação viciosa, necrose avascular, rigidez e aderências. ${ }^{4-6}$

Em 2010, Boulton et al descreveram uma técnica de fixação para fraturas subcapitais dos metacarpos com o objetivo de minimizar estas complicações e ainda promover a mobilidade precoce, utilizando um parafuso canulado sem cabeça com fixação retrógrada. ${ }^{7}$ Mais tarde, Del Piñal et al ${ }^{8}$ aperfeiçoaram a técnica, adicionando diferentes métodos de redução e fixação para fraturas cominutivas e com maior probabilidade de perda de redução ou encurtamento, aumentando a gama de indica- ções para fraturas complexas. Neste estudo, os autores descreveram 69 fraturas em 59 pacientes, inclusive 17 fraturas expostas, sendo tratadas em apenas um tempo. Ele concluiu que este é seu método de escolha para fraturas diafisárias transversas e oblíquas curtas, além de metafisárias dos metacarpos e falanges, sendo contraindicado apenas em casos de fise aberta e infecção vigente.

Nosso trabalho descreve a técnica cirúrgica e os resultados clínicos da fixação percutânea intramedular retrógrada de fraturas do colo de metacarpos. Em todos os casos foi utilizado um parafuso canulado sem cabeça, numa série de casos operados entre 2015 e 2018.

\section{Métodos}

Após aprovação pelo Comitê de Ética em Pesquisa, foi realizada uma avaliação retrospectiva de dados clínicos e radiográficos de 24 pacientes com fraturas fechadas do colo do metacarpo tratadas cirurgicamente com parafusos autocompressivos sem cabeça (Herbert) retrógrados entre abril de 2015 e novembro de 2018. Três pacientes perderam seguimento e foram excluídos da avaliação. Todos os pacientes foram operados e seguidos pelo autor sênior (Folberg C. R.).

Radiografias padrão em incidências anteroposterior e oblíqua da mão foram obtidas para avaliação do desvio e da angulação das fraturas. A medida da angulação em graus foi medida nas incidências oblíquas. As indicações cirúrgicas 
foram angulação colo-diáfise do metacarpo $>30^{\circ}$ para os II e III metacarpos e $>40^{\circ}$ para o $\mathrm{V}$ metacarpo, encurtamento $\geq 5 \mathrm{~mm}$, desvio rotacional e desejo do paciente de não utilizar imobilização gessada. Todos os pacientes foram avaliados pelo autor sênior tanto no pré- quanto no pósoperatório, sendo discutidos os riscos e benefícios da cirurgia. Antes da assinatura do termo de consentimento informado, além das orientações sobre o procedimento cirúrgico, as instruções pós-operatórias foram amplamente discutidas, explicitando a importância da mobilização precoce sem carga para que pudessem alcançar a mobilidade articular completa o mais brevemente possível.

\section{Técnica cirúrgica}

Todos os pacientes foram operados sob sedação e bloqueio anestésico axilar do plexo braquial, exceto os últimos dois pacientes. Nestes, foi utilizada a técnica Wide Awake Local Anesthesia No Tourniquet (WALANT, na sigla em inglês), quando é utilizada anestesia local com adrenalina e o paciente mantém-se acordado durante o procedimento.

As fraturas eram reduzidas de modo fechado com tração manual, manipulação dos fragmentos proximal e distal e flexão da articulação metacarpofalangeana. Em três casos, foi necessário o auxílio de um fio de Kirschner para redução da fratura, que era introduzido no foco da fratura fazendo alavanca e mantendo a redução temporariamente até que o parafuso de Herbert fosse introduzido.

Uma agulha 16 gauge era posicionada, com auxílio do intensificador de imagens, no centro da cabeça do metacarpo tanto na incidência anteroposterior quanto perfil e direcionada para o canal intramedular. Esta agulha era introduzida manualmente no canal intramedular do metacarpo até cruzar o foco da fratura no fragmento proximal. O fio-guia do parafuso de Herbert era introduzido através da agulha até a base do metacarpo, onde era então fixado (- Figura 1). A agulha era removida e uma incisão (tipo estocada com bisturi no sentido longitudinal do tendão) de $\sim 3 \mathrm{~mm}$ adjacente ao fio-guia era feita através da pele, do tendão extensor e da cápsula articular. Após, eram utilizadas as brocas longa de $1.6 \mathrm{~mm}$ e curta de $2.0 \mathrm{~mm}$ para preparar para a inserção do parafuso de Herbert de $3.0 \mathrm{~mm}$. Em todos os casos, o parafuso mais longo disponível (30 mm) foi utilizado, permanecendo sempre abaixo da superfície articular sob controle radioscópico.

Em 2 pacientes com fraturas cominutivas instáveis, onde a compressão dos fragmentos não seria benéfica para a fixação - o objetivo seria apenas a estabilização da fratura -, um fio de Kirschner de $1.5 \mathrm{~mm}$ foi passado transfixando e estabilizando o fragmento distal ao metacarpo adjacente. Após a estabilização da fratura com o parafuso, mantendo a altura do metacarpo, o fio de Kirchner era retirado. Nos demais casos, o desvio rotacional era controlado visualizando a fluoroscopia e ao terminar o procedimento com mobilidade ativa ou passiva dos dedos. Não houve necessidade de fixação temporária do fragmento distal para controle rotacional, pois durante a fixação não pareceu haver desvio rotacional significativo. Desvios rotacionais e estabilidade da osteossíntese eram testados logo após a fixação. Nos pacientes nos quais foi utilizada anestesia local (WALANT), era realizado o teste com mobilidade ativa, e nos demais com mobilidade passiva dos dedos e punho.

Após sutura da pele e curativo, uma faixa elástica era aplicada e o paciente era orientado a mobilizar os dedos conforme tolerância por 5 a 7 dias até a primeira revisão pós-operatória, quando radiografias de controle eram obtidas. Com a diminuição da dor e do edema, os pacientes eram encorajados a mobilizar os dedos objetivando amplitude de movimento completa. Na consulta seguinte, em torno de 3 semanas pósoperatórias, novas radiografias eram realizadas. Se a amplitude de movimento estivesse melhorando muito lentamente, o paciente era encaminhado para clínica de fisioterapia. Do contrário, era estimulado a manter os exercícios em casa. Os pacientes eram reavaliados com 6 e 12 semanas pós-operatórias, quando a maioria dos pacientes tinha sua avaliação final se apresentassem mobilidade ampla e ausência de queixas.

\section{Resultados}

A casuística incluiu 19 homens e 2 mulheres, com média de idade de 33,4 anos (18-75 anos). A fratura ocorreu na mão direita em 15 pacientes e na mão esquerda em 6 pacientes. Os mecanismos causadores do trauma foram soco contra parede/mesa/outrem $(n=14)$, queda ao solo $(n=5)$ e acidente de trânsito $(\mathrm{n}=2)$. As fraturas ocorreram no colo do quinto metacarpo $(n=19)$, do terceiro metacarpo $(n=1)$ e do segundo metacarpo $(\mathrm{n}=1)$.

O tempo médio decorrido da fratura até a cirurgia foi de $9 \pm 6$ dias.

Os dados dos pacientes estão resumidos na - Tabela 1.

O tempo operatório em todos os casos foi $<45$ minutos (intervalo entre 10 e 42 minutos). Não houve nenhuma complicação transoperatória.

Todas as fraturas apresentaram consolidação clínica e radiológica. Não houve nenhum caso de consolidação viciosa (nenhum desvio angular ou rotacional significativo). As últimas radiografias obtidas com seguimento mais longo não mostraram alterações degenerativas em nenhum paciente.

Nenhum paciente teve infecção pós-operatória, aderências tendíneas ou capsulares ou complicações relacionadas ao parafuso que necessitassem reoperação. Todos os 21 pacientes tiveram um resultado funcional excelente, com amplitude de movimento total ativa do dedo $>240^{\circ}$. Apenas um paciente (fratura do segundo metacarpo) teve um lag extensor de $10^{\circ} \mathrm{e}$ flexão da articulação metacarpofalangeana $10^{\circ}$ menor que o lado contralateral (-Figura 2), sem repercussão funcional. Dois pacientes tiveram que permanecer fazendo fisioterapia e terapia ocupacional após 12 semanas de pós-operatório até obter amplitude de movimento completa, um com 16 e outro com 20 semanas. Todos os pacientes voltaram às suas atividades prévias, tanto laborativas quanto esportivas e recreacionais, sem queixas em relação à função das mãos.

\section{Discussão}

O uso de fios de Kirschner no tratamento cirúrgico das fraturas isoladas desviadas de metacarpos foi, por muito tempo, o método de escolha. Baseado nos conceitos de 

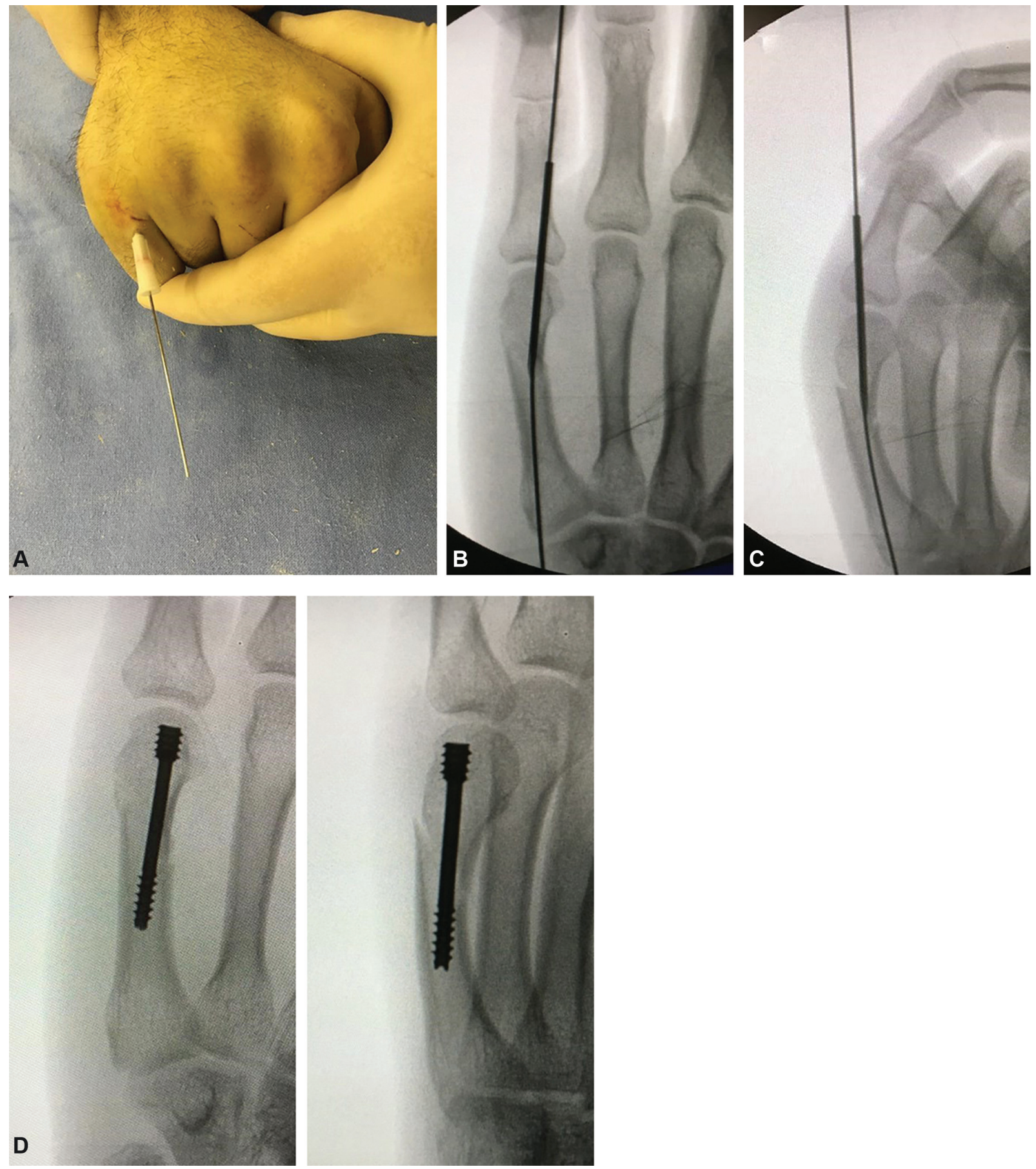

Fig. 1 Detalhes da técnica cirúrgica: A) Introdução da agulha e fio-guia após redução da fratura; B e C) Controle radioscópico; D e E) Resultado pós-operatório imediato da fixação.

fixação flexível proposto por Ender et $\mathrm{al}^{9} \mathrm{e}$, posteriormente, por Foucher, ${ }^{10}$ com a osteossíntese intramedular anterógrada, ou estabilizando a fratura com fios cruzados, apresenta bons resultados. ${ }^{11}$ Todavia, complicações como infecção, perda precoce da fixação, rigidez e não-consolidação podem chegar a $16 \%$ dos casos. ${ }^{12,13}$

A escolha por parafusos canulados autocompressivos sem cabeça introduzida por Herbert et al para tratamento de fraturas do punho e do carpo, ${ }^{14}$ e mais recentemente no tratamento de fraturas de metacarpos e falanges ${ }^{8,15-18}$ é uma alternativa aos tratamentos convencionais que traz algumas vantagens: não viola o foco da fratura, não necessita imobilização gessada e permite mobilidade precoce Além disso, diversos estudos mostraram superioridade biomecânica dos parafusos intramedulares em relação aos fios de Kirschner intramedulares. ${ }^{19,20}$ A estabilidade no foco da fratura pode ser dada pela fixação da rosca do parafuso no canal endosteal ou como tutor intramedular, podendo inclusive ser um misto de ambos, como sugerido por Del Piñal et al. ${ }^{8}$

Nossa técnica propõe a colocação do fio-guia sem incisão prévia, e apenas uma incisão mínima (tipo estocada com a lâmina do bisturi no sentido longitudinal do tendão), não 
Tabela 1 Caracterização da amostra

\begin{tabular}{|l|l|}
\hline Variáveis & $\mathbf{n}=\mathbf{2 1}$ \\
\hline Idade (anos) - média \pm DP & $33,5 \pm 14.3$ \\
\hline Lado acometido - n (\%) & \\
\hline Direito & $15(71,4)$ \\
\hline Esquerdo & $6(28,6)$ \\
\hline Osso - n (\%) & \\
\hline II Metacarpo & $1(4,8)$ \\
\hline III Metacarpo & $1(4,8)$ \\
\hline V Metacarpo & $19(90,5)$ \\
\hline Angulação (graus) - média \pm DP & $32,0 \pm 0,0$ \\
\hline II Metacarpo & $35,0 \pm 0,0$ \\
\hline III Metacarpo & $49,8 \pm 11,4$ \\
\hline V Metacarpo & \\
\hline Mecanismo - n (\%) & $14(66,7)$ \\
\hline Soco & $5(23,8)$ \\
\hline $\begin{array}{l}\text { Queda ao solo (futebol, bicicleta, } \\
\text { queda da própria altura) }\end{array}$ & $2(9,5)$ \\
\hline $\begin{array}{l}\text { Acidente moto/carro } \\
\text { Tempo de lesão até cirurgia } \\
\text { (dias) - média } \pm \text { DP }\end{array}$ & $9,14 \pm 6,17$ \\
\hline
\end{tabular}

Abreviação: DP, desvio-padrão.

havendo visualização direta de tendão, da cápsula articular e da superfície articular do metacarpo. Ruchelsman et al $^{16}$ descreveram uma pequena abordagem com abertura longitudinal do tendão extensor e artrotomia dorsal, mas, assim como o trabalho de Jann et $\mathrm{al}^{17}{ }^{17}$ acreditamos que a fluoroscopia fornece uma boa visualização do ponto de entrada do parafuso. A lesão do tendão extensor, assim como a lesão condral da cabeça do metacarpo, causadas pela entrada da cabeça do parafuso, não foram consideradas significativas. ${ }^{18,21}$ A solicitação da mobilização ativa da mão, com extensão completa do dedo logo após a passagem do parafuso, demonstrou funcionalidade do mecanismo extensor no transoperatório. Isso se manteve desta forma em todos os casos. A fixação dessas fraturas com anestesia local com adrenalina e sem uso do torniquete (WALANT, como descrito por Lalonde et al, ${ }^{22}$ oportuniza que o paciente acordado faça mobilidade ativa no transoperatório. Isso possibilita testar o mecanismo extensor e visualizar a estabilidade da osteossíntese sob fluoroscopia, reforçando para o paciente a ideia de poder mobilizar o dedo precocemente com segurança. A proteção do dedo afetado sindactilizando-o com o dedo adjacente por 30 dias no pósoperatório como realizado no trabalho de Jann et al ${ }^{17}$ não se mostrou necessária em nossos casos.

Alguns autores afirmam não haver compressão no foco de fratura, pois o parafuso funcionaria apenas como um estabilizador interno. ${ }^{23}$ Entretanto, nas fraturas mais cominutivas, a compressão do parafuso pode instabilizar a fratura e ocasionar desvio e encurtamento da fratura. Nestes casos, o uso de um segundo parafuso (via de regra de diâmetro menor) para estabilização está indicado, conforme bem descrito por Del Piñal et al, ${ }^{8}$ mas exige maior habilidade do cirurgião, pois o espaço muitas vezes é pequeno para colocação de dois parafusos. A colocação de um fio de Kirchner de $1,5 \mathrm{~mm}$ entre as cabeças do metacarpo fraturado e a adjacente, volarmente ao parafuso já parcialmente penetrado, nos pareceu ser fácil e prático. Esta manobra impede que o parafuso faça compressão no foco da fratura, mas permite uma ótima estabilidade ao fixar o parafuso na cabeça. Nos dois casos em que utilizamos essa técnica, retiramos o fio logo após passar o parafuso, confirmamos a estabilidade do foco da fratura e obtivemos bons resultados.

Os resultados da nossa série de pacientes são condizentes com os da literatura, especialmente com os trabalhos de Del Piñal et al, ${ }^{8}$ Ruchelsman et al, ${ }^{16}$ Tobert et al, ${ }^{23}$ Doarn et al $^{24} \mathrm{e}$ Romo-Rodriguez et al, ${ }^{25}$ todos apresentando ótimos resultados funcionais e nenhum caso de pseudoartrose ou consolidação viciosa. Mais recentemente, Beck et al reforçam estes achados em sua revisão da literatura sobre o tema. ${ }^{26} \mathrm{Em}$ um paciente da nossa série, um pequeno lag extensor $\left(10^{\circ}\right)$ permaneceu, porém esta alteração não foi significativa em relação à amplitude total de movimento $\left(240^{\circ}\right)$, não impedindo um ótimo resultado funcional.

Esta é uma técnica que viola a cartilagem articular da cabeça do metacarpo, sendo passível de críticas. Porém, uma análise quantitativa tridimensional por tomografia computadorizada (TC) realizada por ten Berg et $\mathrm{al}^{21}$ demonstrou que o ponto de entrada dorsal do parafuso na cabeça do metacarpo só é atingido pela base da falange proximal após $87 \%$ do arco de $120^{\circ}$ de extensão realizado no plano sagital. Essa análise também demonstra que a área de superfície da cabeça do metacarpo e o volume subcondral da cabeça ocupados são mínimos. Um estudo mostra que, em médio prazo, não foram encontradas alterações na cabeça de metacarpos com o uso de dispositivos intramedulares de até $5 \mathrm{~mm}$ de diâmetro. ${ }^{27}$ Além disso, vale sempre lembrar do uso já consagrado desse parafuso em escafoide, cabeça de rádio, capitelo e outras superfícies articulares. Não há relato na literatura de complicação por alteração condral em cabeça de metacarpo pós-osteossíntese com parafuso intramedular sem cabeça a curto e médio prazo. Apesar das evidências atuais apontarem para um futuro sem alterações condrais com o uso desta técnica, estudos de longo prazo são necessários para sua comprovação.

Nosso trabalho apresenta algumas limitações: trata-se de um estudo retrospectivo com um número limitado de pacientes. Além disso, o tempo de seguimento de alguns pacientes foi relativamente curto para que se possa chegar a conclusões definitivas sobre alterações degenerativas articulares ou alguma outra complicação que possa surgir com um seguimento mais longo. Mesmo assim, pelos bons resultados aqui apresentados e que reproduzem os achados da literatura, esta técnica confirma ser muito eficaz, segura e reprodutível para osteossíntese de colo de metacarpo.

\section{Conclusão}

A osteossíntese intramedular retrógrada com parafuso sem cabeça (tipo Herbert) nas fraturas do colo do metacarpo apresentou ótimos resultados radiológicos e funcionais em 

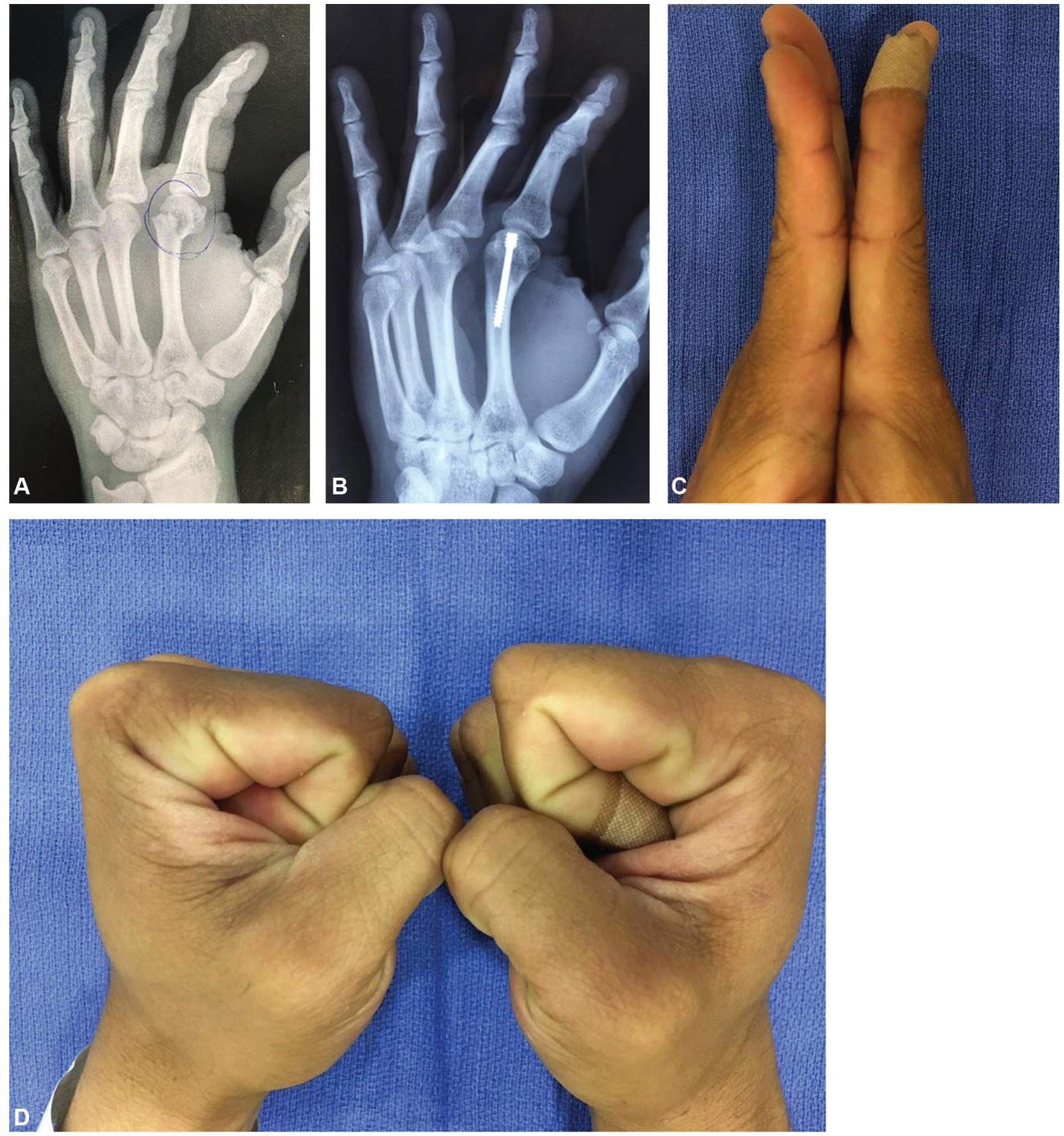

Fig. 2 A) Fratura do colo do segundo metacarpo; B) Radiografia pós-operatória tardia; C) Mínimo lag extensor; D) Leve déficit de flexão da articulação metacarpofalangeana.

todos os pacientes nessa série de 21 casos apresentados e mostrou ser uma excelente opção minimamente invasiva para o tratamento destas fraturas na mão.

\section{Conflito de Interesses}

Os autores declaram não haver conflito de interesses.

\section{Referências}

1 Chung KC, Spilson SV. The frequency and epidemiology of hand and forearm fractures in the United States. J Hand Surg Am 2001; 26(05):908-915

2 Strub B, Schindele S, Sonderegger J, Sproedt J, von Campe A, Gruenert JG. Intramedullary splinting or conservative treatment for displaced fractures of the little finger metacarpal neck? A prospective study. J Hand Surg Eur Vol 2010;35(09):725-729

3 Johnson KA. Posterior tibial tendon. In: Weiss AP, Goldfarb CA, Hentz VR, Raven RB, Slutsky DJ, Steinmann SP, Budoff JE, Calfee $R P$, eds. Textbook of hand and upper extremity surgery. Chicago: American Society for the Surgery of the Hand; 2013:76-90

4 Creighton JJ Jr, Steichen JB. Complications in phalangeal and metacarpal fracture management. Results of extensor tenolysis. Hand Clin 1994;10(01):111-116

5 Kollitz KM, Hammert WC, Vedder NB, Huang JI. Metacarpal fractures: treatment and complications. Hand (N Y) 2014;9(01):16-23

6 Page SM, Stern PJ. Complications and range of motion following plate fixation of metacarpal and phalangeal fractures. J Hand Surg Am 1998;23(05):827-832 
7 Boulton CL, Salzler M, Mudgal CS. Intramedullary cannulated headless screw fixation of a comminuted subcapital metacarpal fracture: case report. J Hand Surg Am 2010;35(08):1260-1263

8 del Piñal F, Moraleda E, Rúas JS, de Piero GH, Cerezal L. Minimally invasive fixation of fractures of the phalanges and metacarpals with intramedullary cannulated headless compression screws. J Hand Surg Am 2015;40(04):692-700

9 Ender J, Simon-Weidner R. Die Fixierung der trochanteren Bruche mit runden elastischen Condylennageln. Acta Chir Austriaca 1970;2 (01):40-42

10 Foucher G. "Bouquet" osteosynthesis in metacarpal neck fractures: a series of 66 patients. J Hand Surg Am 1995;20(3 Pt 2):S86-S90

11 Schädel-Höpfner M, Wild M, Windolf J, Linhart W. Antegrade intramedullary splinting or percutaneous retrograde crossed pinning for displaced neck fractures of the fifth metacarpal? Arch Orthop Trauma Surg 2007;127(06):435-440

12 Hsu LP, Schwartz EG, Kalainov DM, Chen F, Makowiec RL. Complications of K-wire fixation in procedures involving the hand and wrist. J Hand Surg Am 2011;36(04):610-616

13 Stahl S, Schwartz O. Complications of K-wire fixation of fractures and dislocations in the hand and wrist. Arch Orthop Trauma Surg 2001;121(09):527-530

14 Herbert TJ, Fisher WE. Management of the fractured scaphoid using a new bone screw. J Bone Joint Surg Br 1984;66(01):114-123

15 Geissler WB. Cannulated percutaneous fixation of intra-articular hand fractures. Hand Clin 2006;22(03):297-305, vi

16 Ruchelsman DE, Puri S, Feinberg-Zadek N, Leibman MI, Belsky MR. Clinical outcomes of limited-open retrograde intramedullary headless screw fixation of metacarpal fractures. J Hand Surg Am 2014;39(12):2390-2395

17 Jann D, Calcagni M, Giovanoli P, Giesen T. Retrograde fixation of metacarpal fractures with intramedullary cannulated headless compression screws. Hand Surg Rehabil 2018;37(02):99-103
18 Borbas P, Dreu M, Poggetti A, Calcagni M, Giesen T. Treatment of proximal phalangeal fractures with an antegrade intramedullary screw: a cadaver study. J Hand Surg Eur Vol 2016;41(07):683-687

19 Avery DM 3rd, Klinge S, Dyrna F, et al. Headless compression screw versus Kirschner wire fixation for metacarpal neck fractures: a biomechanical study. J Hand Surg Am 2017;42(05):392. e1-392.e6

20 Jones CM, Padegimas EM, Weikert N, Greulich S, Ilyas AM, Siegler S. Headless screw fixation of metacarpal neck fractures: a mechanical comparative analysis. Hand (N Y) 2019;14(02):187-192

21 ten Berg PW, Mudgal CS, Leibman MI, Belsky MR, Ruchelsman DE. Quantitative 3-dimensional CT analyses of intramedullary headless screw fixation for metacarpal neck fractures. J Hand Surg Am 2013;38(02):322-330.e2

22 Lalonde D, Eaton C, Amadio P, Jupiter J. Wide-awake hand and wrist surgery: a new horizon in outpatient surgery. Instr Course Lect 2015;64:249-259

23 Tobert DG, Klausmeyer M, Mudgal CS. Intramedullary Fixation of Metacarpal Fractures Using Headless Compression Screws. J Hand Microsurg 2016;8(03):134-139

24 Doarn MC, Nydick JA, Williams BD, Garcia MJ. Retrograde headless intramedullary screw fixation for displaced fifth metacarpal neck and shaft fractures: short term results. Hand (N Y) 2015;10(02): 314-318

25 Romo-Rodríguez R, Arroyo-Berezowsky C. Osteosíntesis mínimamente invasiva con tornillos centromedulares canulados para fracturas de metacarpianos. Acta Ortop Mex 2017;31 (02):75-81

26 Beck CM, Horesh E, Taub PJ. Intramedullary screw fixation of metacarpal fractures results in excellent functional outcomes: a literature review. Plast Reconstr Surg 2019;143(04):1111-1118

27 Evrard H, Nokerman B. [Centromedullary nailing in metacarpal fractures]. Acta Orthop Belg 1973;39(06):1035-1044 